\title{
Coronary-aortic interaction during ventricular isovolumic contraction
}

\author{
Marc J. van Houwelingen • Daphne Merkus • \\ Maaike te Lintel Hekkert · Geert van Dijk • \\ Arnold P. G. Hoeks • Dirk J. Duncker
}

Received: 15 December 2010/Accepted: 23 March 2011/Published online: 13 April 2011

(C) The Author(s) 2011. This article is published with open access at Springerlink.com

\begin{abstract}
In earlier work, we suggested that the start of the isovolumic contraction period could be detected in arterial pressure waveforms as the start of a temporary presystolic pressure perturbation $\left(\mathrm{AIC}_{\text {start }}\right.$, start of the Arterially detected Isovolumic Contraction), and proposed the retrograde coronary blood volume flow in combination with a backwards traveling pressure wave as its most likely origin. In this study, we tested this hypothesis by means of a coronary artery occlusion protocol. In six Yorkshire $\times$ Landrace swine, we simultaneously occluded the left anterior descending (LAD) and left circumflex (LCx) artery for $5 \mathrm{~s}$ followed by a 20-s reperfusion period and repeated this sequence at least two more times. A similar procedure was used to occlude only the right coronary artery (RCA) and finally all three main coronary arteries simultaneously. None of the occlusion protocols caused a decrease in the arterial pressure perturbation in the aorta during occlusion $(P>0.20)$ nor an increase during reactive hyperemia $(P>0.22)$, despite a higher deceleration of coronary blood volume flow $(P=0.03)$ or increased coronary conductance $(P=0.04)$ during hyperemia. These
\end{abstract}

M. J. van Houwelingen $(\bowtie) \cdot D$. Merkus .

M. te Lintel Hekkert - D. J. Duncker

Experimental Cardiology, Thoraxcenter, Cardiovascular

Research Institute COEUR, Erasmus MC, University Medical

Center Rotterdam, Dr Molewaterplein 50, P.O. Box 2040,

3000 CA Rotterdam, The Netherlands

e-mail: m.vanhouwelingen@erasmusmc.nl

G. van Dijk

MACAWI b.v., Eindhoven, The Netherlands

A. P. G. Hoeks

Biomedical Engineering, Cardiovascular Research Institute

Maastricht (CARIM), Maastricht University, Maastricht,

The Netherlands results show that the pre-systolic aortic pressure perturbation does not originate from the coronary arteries.

Keywords Isovolumic contraction - Coronary-aortic interaction - Coronary occlusion - Pulse wave analysis

\section{Introduction}

The arterial blood pressure waveform and its propagation characteristics contain a vast amount of information on various aspects of cardiovascular structure and function [1, 12], including arterial stiffness $[4,11]$, mechanical function of the heart [3, 18], and the severity of shock [7, 15].

Despite intense examination of the aortic pressure curve, small details are prone to be overlooked or erroneously discarded as noise. In a previous study, we reported the presence of an arterial blood pressure perturbation preceding aortic valve opening in the common carotid artery in a group of healthy subjects as well as in the central aorta in a group of patients following aortic valve replacement [17]. The onset of this perturbation not only correlated in time with the preceding R-top of the ECG, but was also shown to coincide with the onset of the ventricular isovolumic contraction.

The exact cause of the perturbation was not determined in that study, but we hypothesized that the arterial pressure perturbation originated from the coronary arteries. During contraction, blood is almost instantly squeezed out of the myocardial vascular bed forward into the coronary sinus and backward into the coronary arteries. The latter causes a sharp decline in coronary blood volume flow $[3,20]$ or even a retrograde coronary blood volume flow, as well as a small pressure wave, traveling backwards toward the aorta $[5,21]$. Both retrograde coronary blood volume flow and 
coronary pressure waves could contribute to the arterial pressure perturbation occurring prior to aortic valve opening.

In light of these considerations, we set out to test the hypothesis that coronary retrograde blood volume flow in combination with a backwards traveling coronary pressure wave during ventricular isovolumic contraction are the origin of the arterial pre-systolic pressure perturbation. For this purpose, we assessed the pressure perturbation in the aorta in anesthetized swine before, during and after coronary artery occlusion. Specifically, we hypothesized that if the arterial pressure perturbation originates from the coronary artery circulation, it should be abolished by coronary artery occlusion. Conversely, during coronary reactive hyperemia following the release of occlusion the arterial pressure perturbation should be augmented.

\section{Materials and methods}

Experiments were performed in six 3-4 month-old female Yorkshire $\times$ Landrace swine $(44 \pm 12 \mathrm{~kg}$, mean $\pm \mathrm{SD})$ in accordance with the Guide for the Care and Use of Laboratory animals (NIH Publication No. 85-23, revised 1996) and with approval of the Erasmus MC Animal Care Committee.

\subsection{Instrumentation}

Animals were sedated with intramuscular injection of Xylazine $(2.25 \mathrm{mg} / \mathrm{kg})$ and Tiletamine $(5 \mathrm{mg} / \mathrm{kg})$ plus Zolazepam $(5 \mathrm{mg} / \mathrm{kg})$ [10], anesthetized with a bolus of intravenous pentobarbital $(15 \mathrm{mg} / \mathrm{kg})$, intubated and ventilated with a tidal volume of $10 \mathrm{ml} / \mathrm{kg}$. Ventilation rate was adjusted to keep blood gas concentrations within their physiological ranges. Anesthesia was maintained by a continuous pentobarbital infusion (6-12 $\mathrm{mg} / \mathrm{kg} / \mathrm{h} \mathrm{IV}$ ) [19].

A high fidelity lumen pressure sensor catheter (SPC 780C, Millar Instruments, Houston, Texas, USA) was inserted via the right carotid artery and advanced to measure aortic pressure $\sim 2 \mathrm{~cm}$ distal to the aortic valve. Balloon occluders (Fine Science Tools $3 \mathrm{~mm}$ ) were positioned around the left anterior descending (LAD), left circumflex (LCx) and right (RCA) coronary arteries. Coronary blood volume flow probes $(3 \mathrm{~mm}$, Transonic, Ithaca, New York, USA) were placed around the proximal LAD and RCA distal to the occluders for measurement of coronary blood flow. An electromagnetic aortic blood volume flow probe (Skalar, Delft, The Netherlands) was placed around the aorta just distal to the coronary arteries to monitor aortic blood volume flow.

\subsection{Signal recording}

ECG, aortic pressure and aortic and coronary blood volume flow signals were fed into a data acquisition board (National Instruments, Austin, Texas, USA, sample frequency $2000 \mathrm{~Hz}$ ) and stored for offline processing. ECG and pressure transducer signal were amplified using a custom made amplifier system (EMI, Rotterdam, The Netherlands). Matlab (Natick, Massachusetts, USA) was used for storage and automated post processing of the recorded signals.

\subsection{Protocol}

After $30 \mathrm{~min}$ of stabilization, triplicate $25 \mathrm{~s}$ sham measurements of ECG, aortic pressure and LAD and RCA blood volume flows were obtained. Subsequently, the LAD and LCx were simultaneously occluded three times for $5 \mathrm{~s}$ with a minimum interval of $20 \mathrm{~s}$ to allow restoration of coronary blood volume flow to baseline [6]. This procedure was followed by three 5 s occlusions of the RCA separated by $20 \mathrm{~s}$. Finally, all three coronary arteries were simultaneously occluded three times, using the same 5 s occlusion and $20 \mathrm{~s}$ reperfusion intervals.

\subsection{Data analysis}

Measurement equipment introduced a $>10 \mathrm{~ms}$ delay in the ECG compared to the aortic pressure and coronary blood volume flow signals. The delay was compensated for during post processing prior to extracting hemodynamic features. All signals were low pass filtered with a cut-off frequency of $80 \mathrm{~Hz}$ to remove noise. The onset of each heart cycle was identified using the Q-top of the ECG. From the aortic pressure recording, the maximum systolic (SBP), minimum diastolic (DBP), and mean blood pressure (MBP) values were determined. The onset of the arterial pressure perturbation, termed $\mathrm{AIC}_{\text {start }}$ (start of the Arterially detected Isovolumic Contraction, Fig. 1) was identified as the position of the maximum of the second derivative of the aortic blood pressure $\left(d^{2} P / d t_{\max }^{2}\right)$ preceding the aortic valve opening [23]. To quantify the pressure perturbation in the aortic pressure following $\mathrm{AIC}_{\text {start }}$, the pressure perturbation area (PPA) enclosed by the aortic pressure and a tangent was calculated. The tangent started at $\mathrm{AIC}_{\text {start }}$ and ran through the point where it touched the aortic pressure curve again (Fig. 1). The results from the occlusion and reactive hyperemia data were normalized to the five cycle pre-occlusion PPA average.

Mean coronary blood volume flows (CBF) in the LAD and RCA were calculated and divided by MBP to calculate LAD and RCA vascular conductance changes. At the onset of ventricular contraction, coronary blood volume flow decreases sharply. The rate of decrease, i.e., slope of the $\mathrm{CBF}$ signal, can be used in conjunction with coronary 
Fig. 1 Example of an aortic pressure curve with the pressure perturbation enlarged on the right. $\mathrm{AIC}_{\text {start }}$ (start of the arterially-detected isovolumic contraction) is indicated by the dash-dot vertical line and the preceding Q-top of the ECG by a triangle. The enlargement shows the touching tangent (dotted line) and the area between the pressure perturbation and this tangent (PPA)

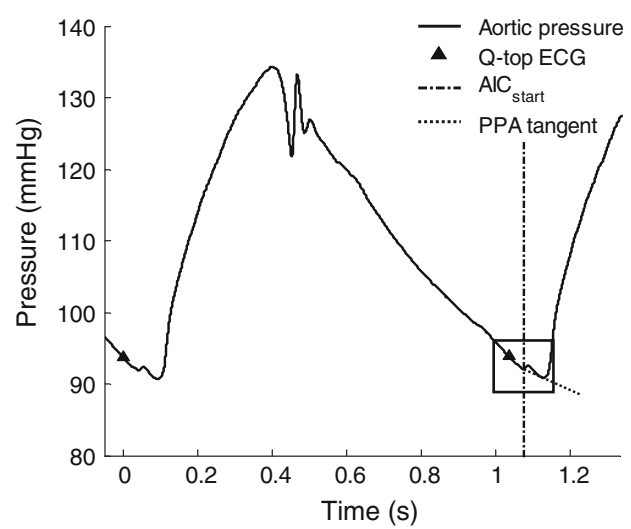

artery pressure for wave intensity analysis [13, 14] to quantify the intensity of the backwards traveling pressure wave during early ventricular contraction. However, coronary artery pressure was not measured in the present study and hence not available. Consequently, the early systolic coronary blood volume flow slope was used as an indicator for wave intensity behavior during early ventricular contraction, assuming that the slope of coronary artery pressure does not change significantly during reactive hyperemia compared to the pre-occlusion slope.

The results of 5 cardiac cycles preceding the onset of occlusion, 5 cycles immediately after the onset of occlusion and 5 cycles following the cessation of occlusion were stored in Excel (Microsoft, Seattle, Washington, USA). The cardiac cycles occurring during the transition from open to fully occluded coronary arteries were excluded from the analysis (typically one to two cycles). Similarly, the cardiac cycles after the release of the occlusion and prior to maximum reactive hyperemia were also excluded from the analysis (typically one to two cycles).

\subsection{Statistics}

All group data are summarized as mean \pm SD. A paired Student's $t$-test was used to test for statistical significance of the changes from baseline to occlusion and from baseline to reperfusion. A $P$ value below 0.05 was considered statistically significant. Statistical analysis of the coronary blood volume flow and aortic pressure data was performed using Excel (Microsoft, Seattle, Washington, USA).

\section{Results}

\subsection{Systemic hemodynamics}

The applied protocols of $5 \mathrm{~s}$ occlusion followed by $20 \mathrm{~s}$ reperfusion did not significantly influence heart rate (HR), SBP, MBP and DBP (Table 1).

\subsection{Effect of occlusion}

Contrary to our hypothesis, no changes in PPA occurred during any of the occlusion combinations (all $P>0.20$, Figs. 2, 3). Furthermore, neither LAD + LCx nor RCA occlusion protocols influenced coronary blood volume flow, vascular conductance or early systolic blood volume flow slope in the non-occluded coronary arteries (Table 1).

\subsection{Effect of reactive hyperemia}

Coronary vasodilatation and reactive hyperemia occurred in the LAD and RCA after release of coronary artery occlusion (Table 1). Compared to baseline values, mean CBF in the LAD and RCA increased by $79 \pm 27 \%$ and $72 \pm 19 \%$, respectively. The steepness of the early systolic blood volume flow slope in both LAD and RCA increased (i.e. the slope became more negative) under influence of reactive hyperemia, although it reached levels of statistical significance only following $\mathrm{LAD}+\mathrm{LCx}$ occlusion $(P=0.03$, Table 1$)$. PPA was not enhanced by reactive hyperemia following any of the occlusion combinations (all $P>0.22$, Fig. 4).

\section{Discussion}

In earlier work, we hypothesized that the arterial pressure perturbation in the aorta preceding aortic valve opening originated from the coronary circulation during contraction. More specifically, we considered retrograde coronary blood volume flow in combination with a pressure wave traveling backwards in the coronary arteries toward the aorta during early systole as cause of the perturbation [23]. Retrograde coronary blood volume flow was first reported by Porter in 1896, and occurs during early ventricular systole [3, 20]. Wave intensity analysis, applied to the waves traveling in the coronary arteries, showed a backwards traveling pressure wave during ventricular 
Table 1 Hemodynamic data

mean $C B F$ mean coronary blood volume flow, $L A D$ left anterior descending coronary artery, $L C x$ left circumflex coronary artery, $R C A$ right coronary artery, $B L$ baseline, $C A O$ coronary artery occlusion, $H$ hyperemia

Values are mean $\pm \mathrm{SD}$

** $P<0.01$ compared to the preocclusion value. $* P<0.05$

indicates a significant difference

compared to the pre-occlusion value

Protocol step

$\mathrm{BL}$

$\mathrm{CAO}$

$\mathrm{H}$

Heart rate (bpm)

sham

$L A D+L C X$

$R C A$

$L A D+L C x+R C A$

Maximum systolic blood pressure $(\mathrm{mmHg})$

$$
\begin{aligned}
& \text { sham } \\
& L A D+L C X \\
& R C A \\
& L A D+L C X+R C A
\end{aligned}
$$

Mean blood pressure $(\mathrm{mmHg})$

$$
\begin{aligned}
& \text { sham } \\
& L A D+L C x \\
& R C A \\
& L A D+L C x+R C A
\end{aligned}
$$

Minimum diastolic blood pressure $(\mathrm{mmHg})$

$$
\begin{aligned}
& \text { sham } \\
& L A D+L C x \\
& R C A \\
& L A D+L C x+R C A
\end{aligned}
$$

\begin{tabular}{|c|c|c|}
\hline $86 \pm 41$ & $86 \pm 41$ & $86 \pm 42$ \\
\hline $90 \pm 43$ & $90 \pm 43$ & $90 \pm 43$ \\
\hline $90 \pm 49$ & $90 \pm 49$ & $90 \pm 49$ \\
\hline $98 \pm 57$ & $98 \pm 57$ & $97 \pm 57$ \\
\hline $118 \pm 23$ & $120 \pm 25$ & $119 \pm 23$ \\
\hline $122 \pm 27$ & $126 \pm 28$ & $121 \pm 24$ \\
\hline $111 \pm 20$ & $114 \pm 20$ & $113 \pm 19$ \\
\hline $114 \pm 25$ & $116 \pm 26$ & $111 \pm 20$ \\
\hline $100 \pm 22$ & $101 \pm 23$ & $101 \pm 21$ \\
\hline $104 \pm 25$ & $106 \pm 26$ & $102 \pm 23$ \\
\hline $93 \pm 18$ & $95 \pm 19$ & $95 \pm 18$ \\
\hline $97 \pm 23$ & $98 \pm 24$ & $94 \pm 20$ \\
\hline $83 \pm 20$ & $83 \pm 21$ & $84 \pm 20$ \\
\hline $86 \pm 24$ & $88 \pm 24$ & $85 \pm 22$ \\
\hline $76 \pm 17$ & $77 \pm 17$ & $78 \pm 16$ \\
\hline $80 \pm 21$ & $82 \pm 21$ & $78 \pm 18$ \\
\hline $52 \pm 16$ & $51 \pm 17$ & $52 \pm 15$ \\
\hline $57 \pm 24$ & - & $105 \pm 53^{*}$ \\
\hline $64 \pm 5$ & $66 \pm 6$ & $64 \pm 6$ \\
\hline $61 \pm 26$ & - & $103 \pm 39^{* *}$ \\
\hline $57 \pm 44$ & $59 \pm 47$ & $57 \pm 45$ \\
\hline $34 \pm 16$ & $34 \pm 16$ & $33 \pm 15$ \\
\hline $68 \pm 45$ & - & $127 \pm 82$ \\
\hline $70 \pm 45$ & - & $114 \pm 74$ \\
\hline $0.54 \pm 0.24$ & $0.53 \pm 0.24$ & $0.54 \pm 0.24$ \\
\hline $0.61 \pm 0.34$ & - & $1.11 \pm 0.68^{*}$ \\
\hline $0.71 \pm 0.25$ & $0.72 \pm 0.26$ & $0.71 \pm 0.26$ \\
\hline $0.67 \pm 0.39$ & - & $1.17 \pm 0.64^{*}$ \\
\hline $0.57 \pm 0.37$ & $0.57 \pm 0.39$ & $0.56 \pm 0.38$ \\
\hline $0.35 \pm 0.06$ & $0.35 \pm 0.05$ & $0.35 \pm 0.06$ \\
\hline $0.70 \pm 0.40$ & - & $1.30 \pm 0.74^{*}$ \\
\hline $0.68 \pm 0.37$ & - & $1.17 \pm 0.64$ \\
\hline$-69.5 \pm 51.1$ & $-71.2 \pm 51.4$ & $-67.5 \pm 52.1$ \\
\hline$-79.5 \pm 37.9$ & - & $-93.7 \pm 43.6^{*}$ \\
\hline$-80.6 \pm 45.4$ & $-81.7 \pm 47.4$ & $-77.6 \pm 44.7$ \\
\hline $83.3 \pm 51.2$ & - & $-97.7 \pm 49.8$ \\
\hline$-57.2 \pm 10.1$ & $-60.9 \pm 8.6$ & $-52.2 \pm 21.0$ \\
\hline$-47.4 \pm 18.8$ & $-46.4 \pm 16.7$ & $-50.6 \pm 5.5$ \\
\hline$-66.3 \pm 29.6$ & - & $-81.6 \pm 40.5$ \\
\hline $79.8 \pm 49.7$ & - & $-97.6 \pm 47.6$ \\
\hline
\end{tabular}

Mean $\mathrm{CBF}_{\mathrm{LAD}}(\mathrm{ml} / \mathrm{min})$

$$
\begin{aligned}
& \text { sham } \\
& L A D+L C X \\
& R C A \\
& L A D+L C x+R C A
\end{aligned}
$$

Conductance $_{\text {LAD }}(\mathrm{ml} / \mathrm{min} / \mathrm{mmHg})$

$$
\begin{aligned}
& \text { sham } \\
& L A D+L C x \\
& R C A \\
& L A D+L C x+R C A
\end{aligned}
$$

Conductance $_{\text {RCA }}(\mathrm{ml} / \mathrm{min} / \mathrm{mmHg})$

$$
\begin{aligned}
& \text { sham } \\
& L A D+L C X \\
& R C A \\
& L A D+L C X+R C A
\end{aligned}
$$

LAD early systole volume flow slope $\left(10^{3} \mathrm{ml} / \mathrm{min}^{2}\right)$

$$
\begin{aligned}
& \text { sham } \\
& L A D+L C X \\
& R C A \\
& L A D+L C X+R C A
\end{aligned}
$$

RCA early systole volume flow slope $\left(10^{3} \mathrm{ml} / \mathrm{min}^{2}\right)$

$$
\begin{aligned}
& \text { sham } \\
& L A D+L C x \\
& R C A \\
& L A D+L C X+R C A
\end{aligned}
$$


Fig. 2 Shown are the aortic pressure and coronary blood volume flow $(\mathrm{CBF})$ in the left anterior descending (LAD) and right common artery (RCA) during three vessel occlusion, with each period in a sequence expanded for one beat to show details. The start and end of occlusion are indicated with a solid vertical line. The vertical dashed lines indicate the Q-top of the ECG for the expanded beats. The ticks on the time axis represent a $1 \mathrm{~s}$ interval

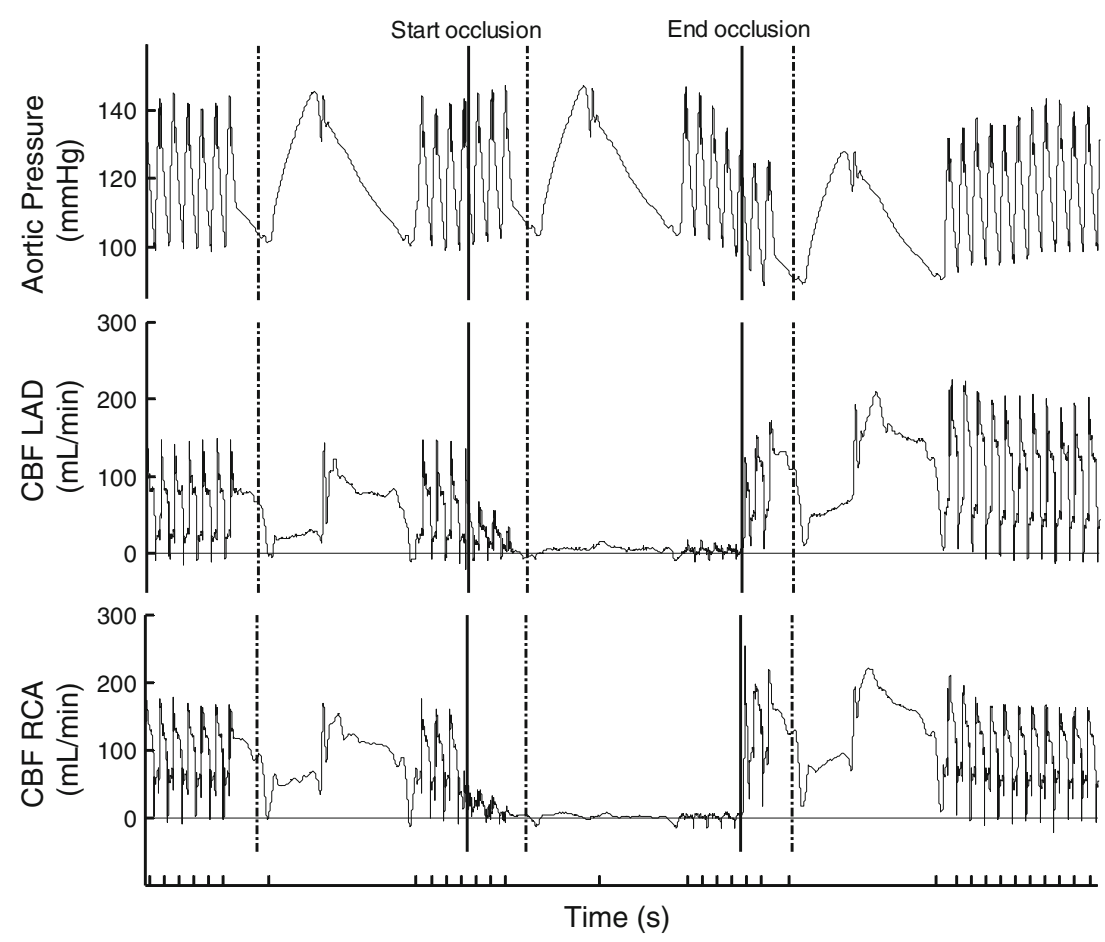

Fig. 3 Pressure perturbation area (PPA) at baseline (BL) and during coronary artery occlusion (CAO). Shown are individual animals (open symbols) and mean \pm SD (solid circles)
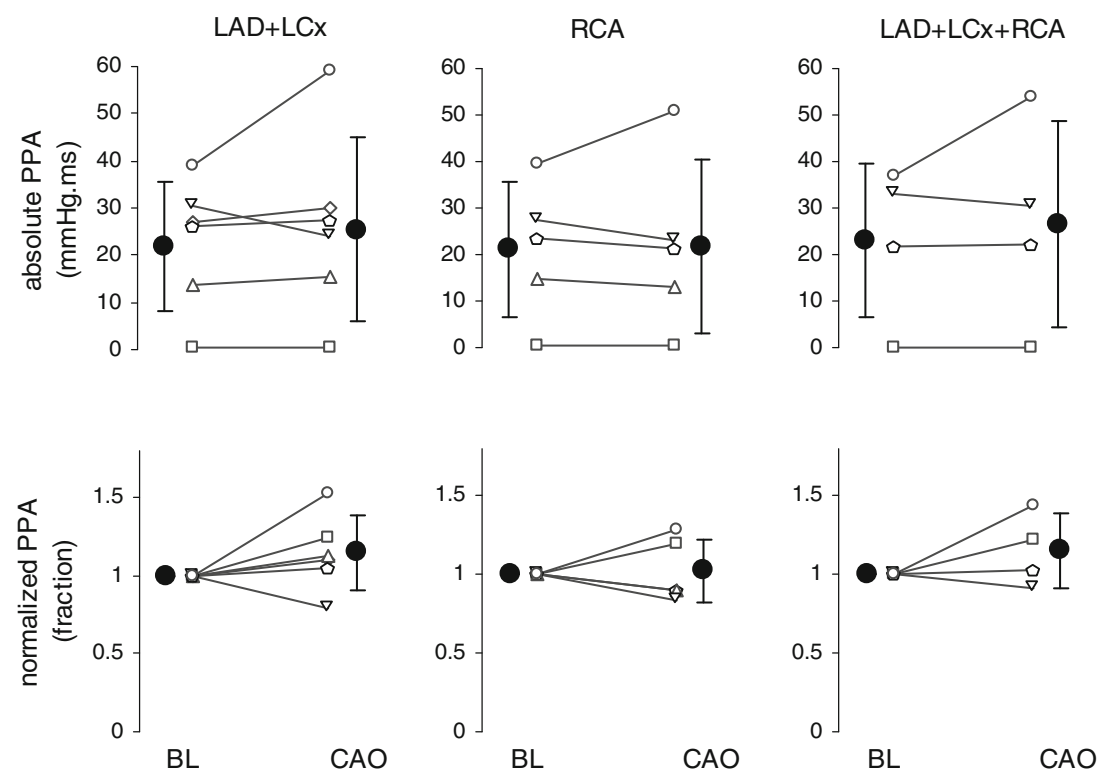

isovolumic contraction [3, 5, 18, 21]. Although not explicitly reported by Davies et al. [5], the presented coronary artery pressure curves contained a clear pressure perturbation with similar timing properties as the investigated backwards compression wave, leading to our hypothesis [23]. However, in this study we found no evidence to support our hypothesis, since the aortic pressure perturbation was not affected by coronary artery occlusion or subsequent hyperemia (respectively, Figs. 3, 4), indicating that the arterial pressure perturbation does not originate from the coronary arteries.

\subsection{Effect of occlusion}

Separate or combined occlusion of the LAD, LCx, and RCA resulted in either a partial or full block of the interaction between the coronary arteries and the aorta. As coronary blood volume flow is blocked, early systolic 
Fig. 4 Pressure perturbation area (PPA) at baseline (BL) and hyperemia $(\mathrm{H})$. Shown are individual animals (open symbols) and mean $\pm \mathrm{SD}$ (solid circles)
LAD+LCx
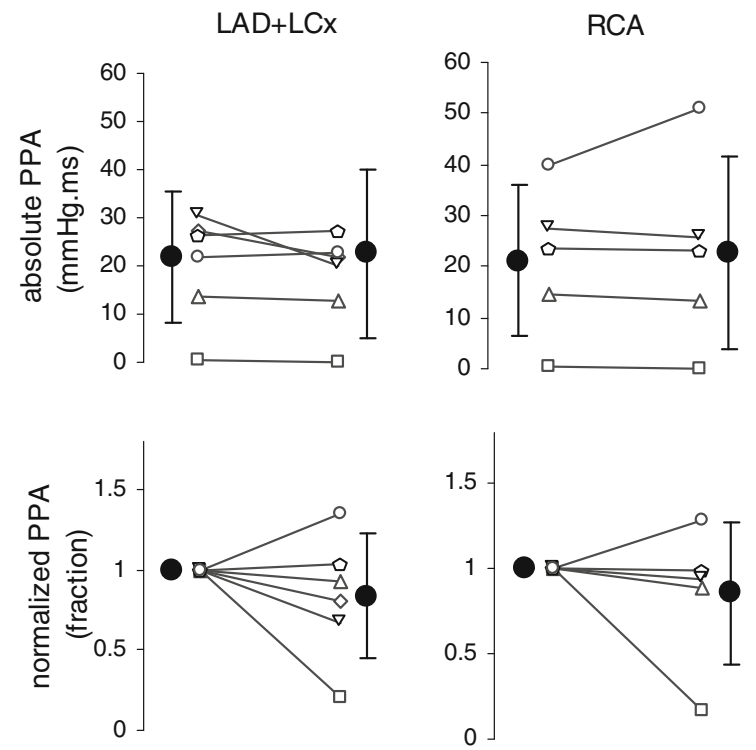

$\mathrm{BL}$

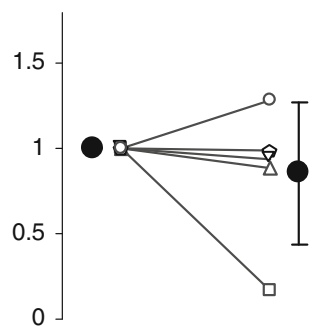

$B L$
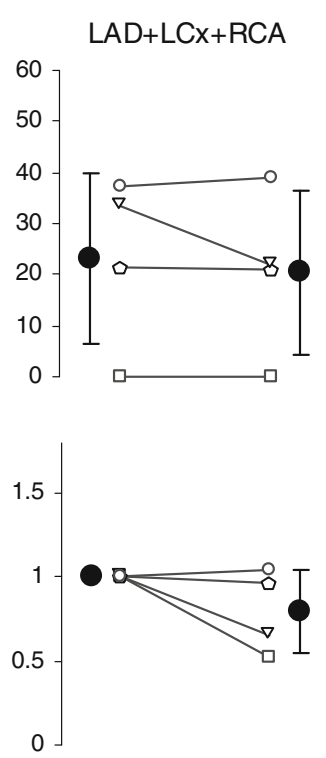

BL retrograde blood volume flow and the backwards traveling coronary pressure waves cannot enter the aorta. If the coronaries are the origin of the arterial pressure perturbation in the aorta that occurs just before aortic valve opening, occluding one or more coronary arteries is expected to decrease the magnitude of the perturbation or to abolish it entirely. Especially the LAD and LCx coronary arteries were expected to dominate the arterial pressure perturbation as the pressure generated in the left ventricle is higher than in the right ventricle resulting in a higher backwards traveling pressure wave in the LAD and LCX as compared to the RCA [8]. Further, the combined LAD + LCx blood volume flow is higher than RCA blood volume flow [16]. Therefore the largest changes in the PPA were expected to occur during LAD + LCx occlusion and the three vessel occlusion. In fact, with a complete three vessel occlusion the aortic pressure perturbation should be absent. In contrast to our initial hypothesis, we did not observe any decrease in pressure perturbation during any of the three occlusion protocols.

\subsection{Effect of reactive hyperemia}

During reactive hyperemia, maximum coronary blood volume flow increased by as much as $140 \%$ (individual data not shown), which is somewhat lower than the $200 \%$ increase after a 5 s occlusion reported in awake dogs [6]. The difference can be the result of either species differences or the use of pentobarbital as an anesthetic agent in the present study which decreases heart rate [2]. Because the heart rates in our animals ( 90 beats per minute) were indeed much lower than the heart rates reported by Duncker et al.
(115 beats per minute, [6]), the oxygen debt build up during occlusion is lower, explaining the lower magnitude of reactive hyperemia [20]. In our animals, the effect of reactive hyperemia on the intensity waves traveling through the coronary arteries could not be calculated, as coronary artery pressure was not measured [13]. However, Sun et al. [22] showed that an approximate doubling of LCx coronary blood volume flow (and a marked decrease in the slope of early systolic blood volume flow) produced by intracoronary adenosine was not accompanied by a marked decrease in the slope of the coronary pressure upstroke. Thus, our results can be interpreted to suggest that the steeper rate of decrease in coronary blood volume flow during early systole was indicative of an increased backwards compression wave. If the arterial pressure perturbation in the aorta indeed originated from the coronary arteries, the increase in wave intensity was expected to increase PPA. However, contrary to our hypothesis PPA values during reactive hyperemia did not show an increase compared to baseline values. These observations are in agreement with the study by Sun et al. [22], in which the magnitude of the aortic pressure perturbation remained apparently unaltered, despite an eightfold increase in LCx peak backward compression wave intensity during vasodilatation. Similarly, they observed that paired pacing increased peak backwards compression wave intensity, without notably affecting the aortic pressure perturbation. It should be acknowledged that the separation of forward and backward waves is not straightforward as it depends on the determined coronary wave speed [13]. However, since Siebes et al. [18] showed that the energy of forward and backward waves minimally varies with wave speed over a wide range of values, possible errors in the 
amplitude of the backwards compression wave as reported by Sun et al. [22] were assumed to be negligible.

It could be argued that loss of retrograde coronary blood volume flow during hyperemia [6, 20], may have opposed the backward-wave induced increases in PPA values, but this is unlikely for several reasons. First, a contribution of retrograde blood volume flow to PPA is not very likely as LAD retrograde blood volume flow in the present study under baseline conditions lasted only $44 \pm 14 \mathrm{~ms}$ with an amplitude of $-34 \pm 37 \mathrm{ml} / \mathrm{min}$ (resulting in a retrograde volume of $15 \pm 16 \mu \mathrm{l}$ ). This low value of retrograde blood volume flow would produce a pressure perturbation of $19 \times$ $10^{-6} \mathrm{~mm} \mathrm{Hg}$, which is negligible compared to the aortic pressure change induced by the stroke volume $(27 \pm 11 \mathrm{ml}$ under baseline conditions). Furthermore, retrograde blood volume flow did not occur in two of the six animals although these two animals did show a clear pressure perturbation. Second, and most important, we observed that simultaneous occlusion of all three coronary arteries (which blocked both coronary retrograde blood volume flow and coronary backward compression waves) had no effect on PPA.

Taken together, the observation that PPA did not change during either occlusion or reactive hyperemia demonstrates that the coronary arteries are not the source of the aortic pressure perturbation.

\subsection{Methodological considerations}

The quantification of the pressure perturbation is sensitive to the shape of the aortic pressure curve which may depend on factors like heart rate, isovolumic contraction period, vascular stiffness, and systemic vascular resistance. Over the course of all three occlusion protocols, variability was low within each animal $(4.4 \mathrm{mmHg} \mathrm{ms}$ over five consecutive heart cycles at baseline), most likely due to the stable heart rates and blood. In contrast, we observed a large variability in the absolute values of PPA over the entire animal group $(22.3 \pm 13.7 \mathrm{mmHg} \mathrm{ms}$ over five consecutive heart cycles at baseline). For one animal, PPA was a factor 10 smaller compared to the PPA values of the other animals. The heart rate of this particular animal was high (167 beats per minute), thereby shortening ventricular pressure rise time [9]. This might have contributed to a shift of the arterial systolic pressure upslope toward the pressure perturbation. This shift leaves little room between the aortic pressure curve and its touching tangent, thereby decreasing the calculated PPA.

\section{Conclusion}

Although we previously have shown that the arterial pressure perturbation does originate from the contraction of the heart [23], the present study shows that it cannot be explained by backflow from the coronary vasculature or a backwards traveling coronary artery pressure wave produced by ventricular contraction. Therefore, the simultaneous occurrence of the backwards traveling pressure wave observed in the coronary arteries and the arterial pressure perturbation in the aorta is merely coincidental [5, 21, 22], but may reflect a common origin of these two phenomena.

Acknowledgments This study was supported by an unconditional grant from Dräger Medical B.V. and a grant from SenterNovem (KWR09134).

Open Access This article is distributed under the terms of the Creative Commons Attribution Noncommercial License which permits any noncommercial use, distribution, and reproduction in any medium, provided the original author(s) and source are credited.

\section{References}

1. Avolio A, Westerhof BE, Siebes M, Tyberg JV (2009) Arterial hemodynamics and wave analysis in the frequency and time domains: an evaluation of the paradigms. Med Biol Eng Comput 47:107-110

2. Baum D, Halter JB, Taborsky GJ Jr, Porte D Jr (1985) Pentobarbital effects on plasma catecholamines: temperature, heart rate, and blood pressure. Am J Physiol 248:E95-E100

3. Bender SB, van Houwelingen MJ, Merkus D, Duncker DJ, Laughlin MH (2010) Quantitative analysis of exercise-induced enhancement of early- and late-systolic retrograde coronary blood flow. J Appl Physiol 108:507-514

4. Cockcroft JR, Wilkinson IB (2002) Arterial stiffness and pulse contour analysis: An age old concept revisited. Clin Sci (Lond) 103:379-380

5. Davies JE, Whinnett ZI, Francis DP, Manisty CH, Aguado-Sierra J, Willson K, Foale RA, Malik IS, Hughes AD, Parker KH, Mayet J (2006) Evidence of a dominant backward-propagating "Suction" wave responsible for diastolic coronary filling in humans, attenuated in left ventricular hypertrophy. Circulation 113:1768-1778

6. Duncker DJ, van Zon NS, Pavek TJ, Herrlinger SK, Bache RJ (1995) Endogenous adenosine mediates coronary vasodilation during exercise after $\mathrm{K}_{\mathrm{ATP}}^{+}$channel blockade. J Clin Invest 95: 285-295

7. Feissel M, Badie J, Merlani PG, Faller JP, Bendjelid K (2005) Pre-ejection period variations predict the fluid responsiveness of septic ventilated patients. Crit Care Med 33:2534-2539

8. Hadjiloizou N, Davies JE, Malik IS, Aguado-Sierra J, Willson K, Foale RA, Parker KH, Hughes AD, Francis DP, Mayet J (2008) Differences in cardiac microcirculatory wave patterns between the proximal left mainstem and proximal right coronary artery. Am J Physiol 295:H1198-H1205

9. Hayward CS, Avolio AP, O'Rourke MF (2002) Arterial pulse wave velocity and heart rate. Hypertension 40:e8-e9; author reply e8-e9

10. Ko JC, Williams BL, Smith VL, McGrath CJ, Jacobson JD (1993) Comparison of telazol, telazol-ketamine, telazol-xylazine, and telazol-ketamine-xylazine as chemical restraint and anesthetic induction combination in swine. Lab Anim Sci 43:476-480

11. Millasseau SC, Ritter JM, Takazawa K, Chowienczyk PJ (2006) Contour analysis of the photoplethysmographic pulse measured at the finger. J Hypertens 24:1449-1456 
12. O’Rourke MF, Gallagher DE (1996) Pulse wave analysis. J Hypertens 14:S147-S157

13. Parker KH (2009) An introduction to wave intensity analysis. Med Biol Eng Comput 47:175-188

14. Parker KH, Jones CJ (1990) Forward and backward running waves in the arteries: analysis using the method of characteristics. J Biomech Eng 112:322-326

15. Pinsky MR (2003) Probing the limits of arterial pulse contour analysis to predict preload responsiveness. Anesth Analg 96: 1245-1247

16. Pitt A, Friesinger GC, Ross RS (1969) Measurement of blood flow in the right and left coronary artery beds in humans and dogs using the 133Xenon technique. Cardiovase Res 3:100-106

17. Reesink KD, Hermeling E, Hoeberigs MC, Reneman RS, Hoeks AP (2007) Carotid artery pulse wave time characteristics to quantify ventriculoarterial responses to orthostatic challenge. J Appl Physiol 102:2128-2134

18. Siebes M, Kolyva C, Verhoeff BJ, Piek JJ, Spaan JA (2009) Potential and limitations of wave intensity analysis in coronary arteries. Med Biol Eng Comput 47:233-239
19. Sorop O, Merkus D, de Beer VJ, Houweling B, Pistea A, McFalls EO, Boomsma F, van Beusekom HM, van der Giessen WJ, VanBavel E, Duncker DJ (2008) Functional and structural adaptations of coronary microvessels distal to a chronic coronary artery stenosis. Circ Res 102:795-803

20. Spaan JA (ed) (1991) Coronary blood flow mechanics, distribution and control. Kluwers Academic Publishers, Dordrecht

21. Sun YH, Anderson TJ, Parker KH, Tyberg JV (2000) Waveintensity analysis: a new approach to coronary hemodynamics. J Appl Physiol 89:1636-1644

22. Sun YH, Anderson TJ, Parker KH, Tyberg JV (2004) Effects of left ventricular contractility and coronary vascular resistance on coronary dynamics. Am J Physiol Heart Circ Physiol 286:H1590 H1595

23. van Houwelingen MJ, Barenbrug PJ, Hoeberigs MC, Reneman RS, Hoeks AP (2007) The onset of ventricular isovolumic contraction as reflected in the carotid artery distension waveform. Ultrasound Med Biol 33:371-378 